\title{
Universal Accretion Growth Using Sandpile Models
}

\author{
Srabani Datta ${ }^{1}$, Ralph Spencer ${ }^{1}$ \& Shane McKie $^{2}$ \\ ${ }^{1}$ Jodrell Bank Centre for Astrophysics ,Turing Building, University of Manchester, \\ Oxford Road, Manchester M13 9PL, United Kingdom \\ email:Srabani.Datta@manchester.ac.uk \\ ${ }^{2}$ Neuroscience \& Pshychiatry Unit, Manchester Royal Infirmary, Stopford Building, Oxford \\ Road, Manchester M13 9PT, United Kingdom
}

\begin{abstract}
The Bak Tang Weisenfeld (BTW) sandpile process is a model of a complex dynamical system with a large collection of particles or grains in a node that sheds load to their neighbours when they reach capacity. The cascades move around the system till it reaches stability with a critical point as an attractor. The BTW growth process shows self-organized criticality (SOC) with power- law distribution in cascade sizes having slope $-5 / 3$. This self-similarity of structure is synonymous with the fractal structure found in molecular clouds of Kolmogorov dimension 1.67 and by treating cascades as waves, scaling functions are found to be analogous to those observed for velocity structure functions in fluid turbulence. In this paper, we show that this is a naturally occuring universal process giving rise to scale - free structures with size limited only by the number of infalling grains. We also compare the BTW process with other sandpile models such as the Manna and Zhang processes. We find that the BTW sandpile model can be applied to a wide range of objects including molecular clouds, accretion disks and perhaps galaxies.
\end{abstract}

Keywords. accretion, accretion disks: molecular processes; turbulence; ISM:clouds; ISM: kinematics and dynamics

\section{Introduction}

Self organised criticality (SOC) is a universal process found in many naturally occuring systems Bak et al.(1988) Such a system evolves under it's own dynamics and reaches a critical state which has been found to be self-similar or fractal. Let us consider points on a network called nodes connected by links or edges representing interactions between the nodes. Two nodes are called adjacent if they are joined by a link and the degree of a node is it's number of neighbours. A network of degree distribution is the sequence $q_{k}: k \geqslant 0$, where $q_{k}$ is the number of nodes with degree k. Once created, the structure of the network does not chnage, so that neighbours will always remain neighbours, giving rise to a rigid or quenched structure. This is called the BTW process. Zhang (1989) proposed a variant to the BTW process where he treated the cascades as a transport problem in quasi units of energy. A single unit of energy could trigger off a set of connected nodes creating a cascade. These 'quanta' of energy cascaded with equal probability and their energy acted as input energy for neighbouring nodes.Once transferred, the energy of the node was reset to zero. Manna (1991) added another variant to the BTW process. The steady state of the system in the Manna process happens when all sites are either occupied or unoccupied.Partcles are distributed randomly having a power law distribution with slope $1.47 \pm 0.10$

\section{Formation of structure by particle accretion in turbulent sytems}

Datta (2003) Datta (2008) studied the geometrical structure of 7 molecular clouds and found them to be fractal with Kolmogorov dimension 1.68. These clouds were different in location but 


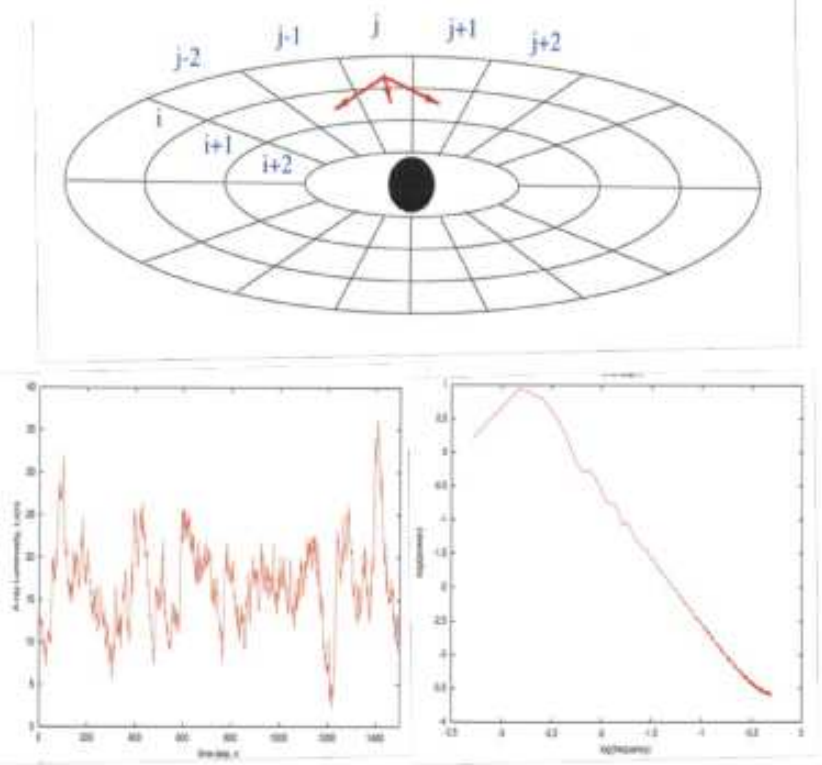

Figure 1. Sandpile processes in the ISM leading to formation of accretion disks. The slope of the X-ray spectrum of Cyg X3 power spectrum is found to be $-1.57 \pm 0.006$

similar in shape to the Julia set given by $z=z_{n}{ }^{2}+c$ where $\mathrm{z}$ and $\mathrm{c}$ are complex quantities and $\mathrm{c}$ $=-0.74543+0.113008 \mathrm{i}$. Kolmogorov proposed that in the region of infinite Reynolds' number, all the statistical properties of a turbulent fluid are universally determined by the scale, mean energy, viscosity and dissipation rate of the system given by a power law of -1.6. McKie (2001) applied the BTW sandpile model to X-ray time series spectra from Cygnus X3 accretion disk and found the gradient to be $-1.57 \pm 0.006$. This implies that this process may be universal in nature underlying the growth seen in a wide range of objects, from accretion disks to molecualr clouds and perhaps even to galaxies.

We thank Prof. T. Mullin for his support during the early phases of this work. We also thank J. Cullen for his assistance with technical issues.

\section{References}

Bak,P.; Tang,C \& Weisenfeld, K. 1988, Phy. Rev. A 38, 364

Datta, S. 2003, AE A 401, 193

Datta, S. 2008, Ph.D. thesis, Calcutta University

Manna, S.S. 1991 Journal of Phys. A 24, L363

McKie, S. 2001 Ph.D. thesis, University of Manchester

Zhang, Y.C. 1989 Phy. Rev. Lett. 63, 470 